\title{
The role of instance retrieval in understanding complex concepts
}

\author{
KENNETH C. GRAY \\ Northwestern University, Evanston, Illinois \\ and \\ EDWARD E. SMITH \\ University of Michigan, Ann Arbor, Michigan
}

\begin{abstract}
Conceptual combination studies have found that a property is often judged more salient in a complex concept than in its constituents. Medin and Shoben (1988), for example, found that metal is more typical than wooden of the simple concept SPOON, whereas wooden is more typical than metal of the complex concept LARGE SPOON. We explored the possibility that such typicality reversals reflect a process of instance retrieval; that is, subjects base their typicality judgments on previously stored instances of LARGE SPOON. This hypothesis predicts that performance in a task requiring typicality judgments and one requiring instance retrieval would be correlated. Three experiments supported this prediction. Experiment 1 provided typicality reversals for a set of concepts. Subjects in Experiments 2 and 3 described instances of the concepts. Aspects of these descriptions were used to predict the reversals. The correlation between predicted and obtained typicality reversals was substantial, and higher for concepts for which subjects retrieved many instances.
\end{abstract}

How do we understand complex concepts like STRIPED BANANA? To the extent that the concept is novel, we must rely on processes of conceptual combination that piece together different bits of existing knowledge into a new conceptual unit. A key question about such processes is whether they are compositional. That is, when combining constituent concepts, like STRIPED and BANANA, into a novel complex concept, like STRIPED BANANA, ${ }^{\prime}$ do the processes of interest use only information about the constituents? Thus far, the research findings appear to argue against compositionality. Springer and Murphy (1992), for example, demonstrated that subjects are faster at verifying a property that is true of a complex concept but not its constituents than a property that is true of both the complex concept and one of its constituents. To illustrate, subjects were able to verify that Boiled celery is soft (soft is a property true of only the complex concept) more quickly than they could verify that Boiled celery is green (green is true of both the simple noun concept and the complex concept). These results indicate that knowledge about the complex concept is more accessible than knowledge about its constituents, which suggests a violation of compositionality.

This kind of argument can be extended. Rather than relying on a property's being true of a complex concept

This research was supported by Air Force Cont ract No. AFOSR-920265 to E. E. Smith. The authors would like to thank Douglas L. Medin and Gregory L. Murphy for comments on an earlier draft of the manuscript. Address correspondence to K. C. Gray, Department of Marketing, J. L. Kellogg Graduate School of Management, Leverone Hall, Northwestern University, Evanston, IL 60208-2001 (e-mail: kgray@ nwu.edu). but not of its constituents, we may say that there is a violation of compositionality whenever a property is judged to be more salient in a complex concept than in its constituents. Using typicality judgments, Medin and Shoben (1988) found evidence for such an effect. For example, they showed that a metal spoon is judged to be more typical of the concept SPOON than is a wooden spoon, whereas a wooden spoon is judged to be more typical of the complex concept LARGE SPOON than is a metal spoon. This typicality reversal indicates that the property wooden is less salient than metal in spoon, but wooden is more salient than metal in large spoon. If conceptual combination is compositional, the salience of nonsize properties (metal and wood) should not change from spoon to large spoon. Note that Springer and Murphy's (1992) results may also be interpreted as a salience reversal: soft is more salient than green in boiled celery, whereas green is more salient than soft in celery (since soft is not even true of celery in general).

\section{Instance Retrieval}

It is possible that the preceding violations of compositionality in conceptual combination are more apparent than real. This is because many of the complex concepts used in the preceding studies may have been familiar and hence not have required any conceptual combination. That is, prior to participating in Springer and Murphy's (1992) study, for example, subjects may have formed the concept BOILED CELERY, and stored it along with some of its instances. Then, when asked to verify Boiled celery is soft, subjects may simply have accessed stored instances of BOILED CELERY and based their decisions on properties of these retrieved instances. The results, then, would re- 
flect aspects of a process of instance retrieval (a memory look-up process), not aspects of conceptual combination (presumably a rule-based process). We will refer to this proposal as the instance retrieval hypothesis. Related ideas have been mentioned by Hampton (1987), Murphy (1988), and Roth and Shoben (1983).

Similar remarks apply to the materials used by Medin and Shoben (1988). LARGE SPOON, for example, may well have been stored in most subjects' mental lexicons prior to the experiment, and if so, subjects may have judged wooden to be typical of LARGE SPOON because many stored instances of LARGE SPOON happen to be wooden. This problem is widespread in the literature on conceptual combination. Most of this research has operationally defined complex concepts as those denoted by more than one word. Although researchers have excluded obviously lexicalized concepts, such as HOUSE BOAT, concepts with which subjects are likely to be familiar fit the operational definition of a complex concept (e.g., LARGE SPOON, PAVED STREET, PEELED APPLE). A quick examination of items from some recent research on conceptual combination reveals that many of the complex concepts under study may, in fact, have been quite familiar to subjects. For example, items used by Murphy (1988) included SLICED APPLES, CASUAL SHIRTS, and SMALL COUCHES. Smith, Osherson, Rips, and Keane (1988) examined complex concepts such as RED FRUIT, ROUND FRUIT, and LONG VEGETABLE. Medin and Shoben (1988) included complex concepts such as PAVED STREET, LARGE BALL, and SMALL BIRD. Springer and Murphy's (1992) experiments included BOILED CELERY and PEELED APPLES, among others. These are not isolated examples. ${ }^{2}$ In short, it is unclear whether the extant evidence for noncompositionality reflects a conceptual combination process or an instance-retrieval process.

In this paper, we provide evidence for the instanceretrieval hypothesis by demonstrating its role in typicality reversals of the sort reported by Medin and Shoben (1988). According to the hypothesis, when asked to judge whether a metal spoon is typical of SPOON, a subject may retrieve instances of SPOON (e.g., the metal spoon I used to put sugar in my coffee), and then check for the property metal among the retrieved instances. When asked to judge if a metal spoon is typical of LARGE SPOON, the subject may retrieve exemplars of LARGE SPOON (e.g., the wooden spoon in the kitchen drawer) and check for the property metal among these instances. An apparent violation of compositionality may arise when the critical property of the retrieved instance for the complex concept differs from that of the retrieved instance for the simple concept. For example, when subjects are judging sPOON, the metal spoon used for coffee is likely to be retrieved but the wooden spoon in the kitchen is not; when subjects are judging LARGE SPOON, however, the kitchen spoon is more likely to be retrieved than the coffee spoon. In essence, an instance-retrieval task is embedded within the typicality judgment. This line of reasoning leads to the basic prediction of the instance-retrieval hypothesis: there should be a correlation between performance in a task requiring typicality judgments about complex concepts and performance in a task that explicitly requires instance retrieval. In addition, we would expect this correlation to be stronger for complex concepts for which subjects can retrieve instances easily. Since familiar complex concepts are often those for which subjects can easily retrieve instances, we might also expect that the correlation would be higher for familiar than for unfamiliar concepts.

The instance-retrieval hypothesis assumes that (part of) a concept's representation consists of stored instances, which means that the hypothesis is in essence an exemplar model of categorization (Medin \& Schaffer, 1978). It has proven difficult in previous research to study exemplar models by using natural categories, but the present experiments are based solely on natural categories and consequently may provide new evidence for exemplar representations (see Heit \& Barsalou, 1992, for additional evidence). In this way, the fate of the instance-retrieval hypothesis has broad implications.

We tested the instance-retrieval hypothesis in three interrelated experiments. In Experiment 1, we obtained typicality judgments for familiar and unfamiliar complex concepts; about half of these judgments revealed typicality reversals. In Experiments 2 and 3, we introduced two instance retrieval tasks, and determined the extent to which results in these tasks could be used to predict the typicality reversals in Experiment 1 . If we were to be successful at predicting typicality reversals, it would suggest that the prior research using many familiar concepts that was interpreted as evidence in favor of noncompositional conceptual combination may have been evidence for instance retrieval instead.

\section{EXPERIMENT 1}

To examine relations between instance retrieval and typicality reversals, first we need to demonstrate some typicality reversals. Experiment 1 was conducted for this purpose. We included items from Medin and Shoben (1988) along with some new ones. Half of the complex concepts used were familiar, and half were unfamiliar. The items were constructed so that approximately equal numbers of reversals and nonreversals would be obtained (based on the results of Medin \& Shoben, 1988, and Smith \& Gray, 1990).

\section{Method}

Materials. Four booklets of items were prepared, each containing the same 101 items but in different orders. ${ }^{3}$ An item consisted of a simple or complex concept-for example, SPOON or LARGE SPOON-with two properties immediately below it-metal and wooden. There were 37 simple concepts (all nouns), 32 familiar complex concepts, and 32 unfamiliar complex concepts. The familiar and unfamiliar complex concepts and properties are given in the first two columns of the Appendix. Each complex concept consisted of one of the simple concepts combined with a modifying adjective or noun. Familiarity determinations were initially 
based on the experimenters' judgments and were then verified by an independent group of subjects. ${ }^{4}$ The items were randomly assigned to pages - 11-12 per page - with the stipulation that a given noun could appear only once per page. Two different assignments of items to pages were used across the four booklets. Also, within each assignment, the left-right placement of properties in an item was randomly determined. However, within a booklet, the position of the properties was held constant for all concepts using the same noun; for example, if metal appeared on the left side for SPOON, it did so for LARGE SPOON and SMALL SPOON as well. Each booklet's pages were shuffled anew for each subject.

Subjects. Twenty-two University of Michigan students participated for pay.

Procedure. The subjects were tested in groups of approximately 5. They were instructed that they would be seeing a number of categories. Below each one, two properties would appear, and their task was to circle the property that was more typical of the category. They were cautioned that some of the categories would be unfamiliar or unusual. Finally, the subjects were encouraged to take their time and to try to think of a reason for their judgments. These instructions were similar to those used by Medin and Shoben (1988)

\section{Results and Discussion}

Consider first the frequency of typicality reversals. If, for example, a subject chose metal as more typical than wooden for SPOON, but wooden as more typical than metal for LARGE SPOON, the choice was scored a reversal. There were 666 reversals and 722 nonreversals $(8$ missing), for a total $48.0 \%$ reversals. This indicates that typicality reversals are widespread. The percentage of subjects who reversed typicality ordering for each complex concept is listed in the third and fourth columns of the Appendix.

Because we used several of Medin and Shoben's (1988) items, we could check whether our results replicated theirs. There were 19 comparisons between a simple concept and a complex one that were common to Medin and Shoben's items and to ours. For the relevant items, we could compare (1) our percent of subjects who reversed with (2) Medin and Shoben's mean typicality ratings for the relevant concepts (Medin and Shoben's task involved ratings rather than choices). If Medin and Shoben's averages showed a reversal and more than $50 \%$ of our subjects reversed in the same direction, we considered it an agreement; if Medin and Shoben's averages showed no reversal and less than $50 \%$ of our subjects reversed, it was also an agreement. Using these criteria, we agreed on 13 of 19 comparisons and disagreed on 6 . Five of the six disagreements were due to the fact that our subjects and Medin and Shoben's subjects gave different typicality orders for the properties for the simple concepts BOOK, WATCH, and STREET. The final disagreement was due to SHORT GRASS (our subjects judged green as more typical than brown, whereas Medin and Shoben's judged brown more typical than green).

If we look at the number of reversals separately for familiar and unfamiliar concepts, we find that 17 of 20 subjects had more reversals for unfamiliar than for familiar complex concepts $(p<.01$ by sign test). One might have expected the opposite - that familiar complex concepts, for which subjects could more easily retrieve instances, would exhibit more typicality reversals than would unfamiliar concepts, for which subjects would more often have to rely on conceptual combination processes. This result suggests that some conceptual combination processes may indeed be noncompositional-an idea to which we will return in the General Discussion. However, as we shall see in Experiment 2, the result does not cause problems for the instance-retrieval hypothesis.

Overall, approximately half of the items exhibited typicality reversals, and they are in reasonable accord with Medin and Shoben (1988) as well as Smith and Gray (1990). We were reasonably confident, then, that we had obtained a reliable set of typicality reversals, similar to those that have been used in previous research to argue that conceptual combination is noncompositional. Our task now was to determine the extent to which these reversals might be due to instance retrieval.

\section{EXPERIMENT 2}

In this experiment, we desired to produce a large number of retrieved instances in order to predict the reversals in Experiment 1. For each of the simple and complex concepts used in Experiment 1, we asked subjects to describe up to four instances. The mention of particular properties-namely, those used in Experiment 1-in these descriptions was used to predict reversals from Experiment 1 . For example, if the instances that subjects retrieved for SPOON were often made of metal, whereas the instances that they retrieved for LARGE SPOON were often made of wood, we predicted that subjects would have often reversed the typicality of metal and wooden when comparing SPOON to LARGE SPOON in Experiment 1 (more precise details are given below).

In order to make our predictions, it was necessary to obtain information only about the target properties for each instance (i.e., the properties from Experiment 1). When subjects were asked to describe instances, however, often they did not spontaneously mention these properties, so we had to ask for them. To prevent subjects from realizing that we were interested in only one piece of information per instance, and thus ensure that they did indeed retrieve instances, we divided the experiment into two parts. In the first part, we asked subjects to retrieve all instances; in the second part, we asked subjects questions about the target properties.

\section{Method}

Materials. Six booklets were constructed; four contained 16 complex concepts each, one contained 19 simple concepts, and the remaining booklet contained 18 simple concepts. All concepts were taken from Experiment 1. Assignment of concepts to booklets was random, except for the following restrictions pertaining to complex concepts: (1) half were familiar and half unfamiliar, (2) half were reversal intended and half were nonreversal intended (according to the typicality ratings of Medin \& Shoben, 1988, 
and Smith \& Gray, 1990), and (3) a given noun appeared only once in a booklet. One random order was used for each type of booklet.

Each booklet was divided into two parts, corresponding to the two parts of the experiment. In the first part of the booklet, each concept was listed at the top of a page with space below it for four instances to be described. At the bottom of the page was a 7-point scale for rating the ease of retrieval for each instance ( 1 was labeled very easy, the instance just popped into my head; 4 was labeled medium difficulty; 7 was labeled very difficult, I thought of the instance only after thinking quite hard). The second part of the booklet contained written questions about the target properties of each instance retrieved in Part 1 (in Part 2, the concepts were listed in the same order as in Part 1). The questions all had two answer choices. For example, for SPOON, the question was, Is it (each of your instances) made out of metal or wood? Four spaces were provided for subjects to answer the question about each instance.

Subjects. A total of 88 University of Michigan undergraduates participated in the experiment for course credit. Each of the four booklets of complex concepts and one booklet of simple concepts was completed by 15 subjects; the remaining booklet of simple concepts was completed by 13 subjects.

Procedure. The subjects were tested in groups of 5 to 15 . Written instructions for Part 1 asked subjects to describe examples or instances of a number of categories. They were told they had $90 \mathrm{sec}$ to describe up to four instances for each category. They were also asked to rate (on the 7-point scale described above) how hard or easy it was to think of each instance. The instructions concluded with mention of several strategies for retrieving or generating in stances, including remembering a specific instance, imagining a prototypical example, thinking of subsets of the category, and constructing an image of the instance. ${ }^{5}$ The written instructions were supplemented with verbal instructions cautioning subjects to describe only one instance at a time and to describe a whole instance rather than a single property of a category (these instructions were intended to prevent common errors made by subjects in a pilot version of this experiment). In Part 2, the subjects were instructed to answer a question for each of the instances retrieved in Part 1. They were informed that the written questions would refer to concepts in the order in which they had appeared in Part 1, so that the subjects could easily check back to their Part 1 responses while answering the questions. The subjects were told to answer "neither" if neither choice was true for an instance.

\section{Results}

Ease of retrieving instances. Given our assumption that the instances described were primarily retrieved from prestored categories (see note 5), we would expect more instances to be retrieved from familiar than from unfamiliar complex concepts. This expectation was confirmed. The mean number of instances retrieved for simple, familiar complex, and unfamiliar complex concepts was 3.45 , 2.76 , and 2.03 , respectively ( 4 was the maximum possible). A one-factor analysis of variance (ANOVA) indicated a significant difference among these three item types $[F(2,98)=160.5, p<.01]$, and subsequent comparisons (using the Tukey HSD procedure) indicated that all pairwise differences were significant at the .05 level. In addition, subjects' ratings indicated that it was easier to retrieve instances of familiar than of unfamiliar complex concepts. The mean ease ratings for the simple, familiar complex, and unfamiliar complex concepts were 2.14, 2.12, and 2.90, respectively (with lower numbers indicating greater ease). Again, an ANOVA revealed a significant difference among these item types $[F(2,98)=$ $35.99, p<.01$ ] , and subsequent comparisons (using the Tukey HSD procedure) indicated that the rating for unfamiliar complex concepts differed significantly from the other ratings.

Prediction of percent reversals for Experiment 1. To predict the percentage of subjects in Experiment 1 who exhibited typicality reversals, as well as the direction of the reversals, we compared the instances retrieved for simple with those for complex concepts. The percentage of instances that had a given property in the simple concept was compared with the percentage that had that same property in the related complex concept. The difference between these percentages was the predicted percentage reversals. For example, suppose that for SPOON, 45 described instances were made of metal and 15 were made of wood, and that for LARGE SPOON, 25 described instances were metal and 25 were wood (recall that each subject could retrieve up to four instances, so totals need not be equal). The difference between these percentages-75\% metal $(45 / 60)$ minus $50 \%$ metal $(25 / 50)$ would be the predicted percentage of reversals for SPOON versus LARGE SPOON. Specifically, $25 \%$ of the subjects in Experiment 1 would be predicted to reverse from saying metal was more typical than wood for SPOON to saying wood was more typical than metal for LARGE SPOON. Predicted percentages of reversals were computed for all comparisons of simple versus complex concept. The percentage of reversals predicted using all instances retrieved and the first instance retrieved by each subject is given in the fifth and sixth columns of the Appendix. The correlations between the predicted percentages and the actual percentage of subjects in Experiment 1 who exhibited the expected typicality reversals were computed. The correlations between predicted and obtained reversals was .58 when all retrieved instances were considered. If we restrict the analysis to only the first instance retrieved by each subject, the correlation was .45 . Both of these correlations are significant $(d f=62, p<$ .01 ), which supports the basic prediction of the instanceretrieval hypothesis.

Recall that this hypothesis further predicts that the correlation between predicted and obtained reversals should be higher for concepts for which instances are easily retrieved (such concepts are more likely to be prestored). If subjects found it easy to retrieve instances for a concept, they should be able to retrieve many (i.e., close to the maximum of four). Accordingly, we divided the concepts into two groups on the basis of how many instances subjects were able to retrieve in Experiment 2 (a median split was used). Using all instances retrieved, the correlation between predicted and observed reversals is .72 for the "many-instances-retrieved" complex concepts versus .35 for the "few-instances-retrieved" complex concepts. The difference between these correlations is significant $(Z=2.06, p<.05)$. When only the first instance retrieved is used to predict reversals, the correlations between predicted and observed reversals for the many-instances-retrieved and few-instances-retrieved 
complex concepts are .62 and .15 , respectively; only the former correlation is significant $(d f=30, p<.01)$. Again, the difference between correlations is significant $(Z=2.17, p<.05)$. These results provide further support for the instance-retrieval hypothesis.

Concepts for which subjects can easily retrieve instances should be familiar. Consequently, we might expect that the correlation between predicted and observed reversals would also be higher for familiar than for unfamiliar concepts. Indeed, the familiarity ratings that we collected are highly correlated with the number of instances that subjects retrieved in Experiment $1(r=.72$; $d f=62, p<.01$ ). If we recompute the correlation between predicted and obtained reversals separately for familiar and unfamiliar concepts (based on a median split), we find that $r=.67$ for familiar versus .54 for unfamiliar concepts. Although this difference between correlations is in the expected direction, it failed to reach an acceptable level of significance $(Z=0.76)$. The correlations between predicted and obtained reversals using the first instance retrieved were $r=.55$ for familiar versus .32 for unfamiliar concepts. Again, the difference is in the expected direction but is not significant $(Z=1.06)$.

The preceding analyses provide support for the instance-retrieval hypothesis. However, it is possible that some of this support is artifactual. Specifically, several concepts exhibited close to $50 \%$ obtained reversals in Experiment 1 and predicted reversals in Experiment 2; while this may indicate that subjects differ widely with respect to the instances they retrieve, it may also be due to the fact that subjects do not understand the task. That is, subjects may be performing more or less randomly for several of the concepts. If this is true, the correlations reported above would be inflated because the concepts for which subjects are confused lead to around $50 \%$ predicted reversals and $50 \%$ obtained reversals. To rule out this possibility, the data were reanalyzed separately for concepts for which subjects exhibited a high level of agreement and concepts for which they did not. Specifically, there were 30 concepts for which more than 15 of the 22 subjects in Experiment 1 agreed with each other about the typicality ordering for the complex concept $(p<.05$ by a sign test). There were 34 concepts for which 15 or fewer subjects in Experiment 1 agreed. The correlations between predicted and obtained reversals for the "high-agreement" concepts were .55 and .37 using all instances retrieved and first instances retrieved, respectively. Both correlations are significant $(d f=28, p<$ .05 ). For the "low-agreement" concepts, the comparable correlations were .57 and .53 using all instances retrieved and first instances retrieved, respectively $(d f=$ $32, p<.05$ ). Thus, it appears that subjects' possible misunderstanding of the task was not a serious problem. However, the fact that the correlation was somewhat lower for the "high-agreement" concepts when the first instance retrieved was used does suggest that some random variation has slightly inflated our observed correlations. Note, however, that the difference between the high- and low-agreement concepts' correlations is not significant $(Z=0.61)$.

Deviations from predicted reversals. One additional fact is apparent from the Appendix. Although the correlations between predicted and obtained reversals are substantial, there appears to be a systematic underprediction. That is, the number of reversals obtained often exceeds the number of reversals predicted. A two-way ANOVA of the frequency of reversals (using familiarity and observed versus predicted as factors) confirms that this observation is correct for unfamiliar concepts. There is a significant main effect of familiarity, such that there are more reversals for unfamiliar than for familiar concepts $[.34$ vs. $.25 ; F(1,124)=4.64, p<.04]$. There is also a main effect of observed versus predicted reversals, such that observed exceeds predicted [.37 vs. .21 ; $F(1,124)=14.75, p<.001]$. There is also a significant interaction $[F(1,124)=4.51, p<.04]$. As can be seen in Figure 1, observed reversals far exceed predicted reversals only for unfamiliar concepts. Thus, we have additional evidence suggesting that instance retrieval plays a significant role for familiar concepts.

The observed deviations from predicted reversals suggest a more direct way to look at the data. That is, we can look at typicality judgments, per se, rather than considering typicality reversals. Specifically, the percentage of retrieved instances that have a particular property in Experiment 2 can be used to predict the number of subjects who judged that property as more typical in Experiment 1 . For example, $85 \%$ of the instances retrieved sing for BIRD in Experiment 2, and 21 out of 22 subjects in Experiment 1 judged singing as more typical than crowing for BIRD. The correlation between the typicality order that is predicted by instance retrieval and the typicality order obtained is quite high $[r=.74 ; d f=99, p<.01]$. For simple concepts, the correlation is .66. For familiar complex concepts and unfamiliar complex concepts, the correlations are .84 and .48 , respectively. Both are significantly greater than $0(d f=30, p<.05)$. In addition, the difference between the correlation for familiar and

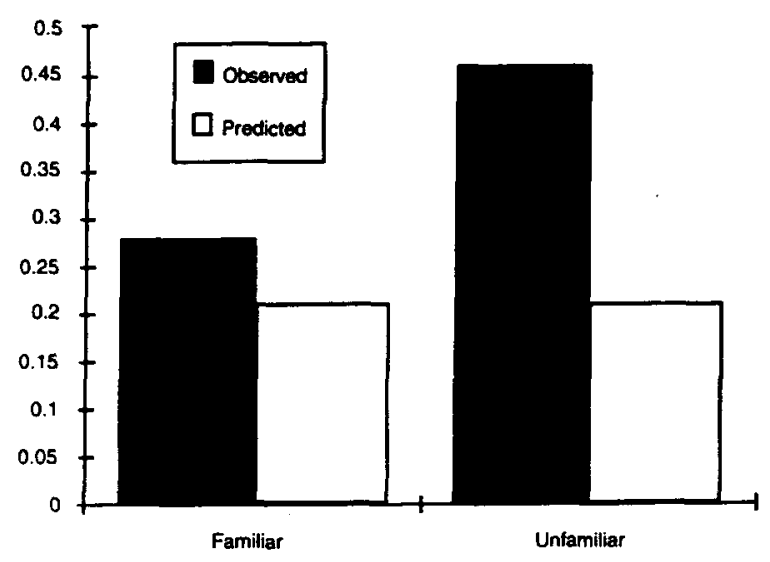

Figure 1. Observed and predicted reversals for familiar and unfamiliar concepts. 
unfamiliar concepts is significant $(Z=2.67, p<.01)$. Thus, we have quite strong evidence for the dependence of typicality judgments (and typicality reversals) on instance retrieval for familiar concepts, or concepts for which subjects can retrieve many instances.

\section{Discussion}

We have shown that the results of an instance-retrieval task can be used to predict the frequency and direction of typicality reversals. As far as we know, this is the first empirical demonstration that the process of instance retrieval plays a role in what were intended to be studies of conceptual combination. Furthermore, we found strong evidence that the dependence of typicality reversals on retrieved instances was greater for those complex concepts for which subjects could retrieve many instances, which provides more support for the instance-retrieval hypothesis. Even when the prediction is based on a single instance per concept (i.e., the first instance generated by each subject), the correlation is higher for the concepts for which subjects could retrieve many instances than for the concepts for which they could retrieve few. Since the complex concepts for which subjects can retrieve many instances also tend to be familiar, the present results suggest that instance retrieval may have played a role in the recent demonstrations of noncompositionality in which somewhat familiar complex concepts were used.

Recall that there were more reversals for unfamiliar than for familiar concepts in Experiment 1 . This result seems at first blush to be at odds with the instanceretrieval hypothesis. But further thought indicates that there is no incompatibility between this result and the instance-retrieval hypothesis. The hypothesis claims only that with more familiar complex concepts, typicality judgments are more likely to be based on instance retrieval (because the instances are easy to retrieve). Although we have emphasized cases in which instance retrieval leads to typicality reversals, there will be many cases in which instance retrieval leads to nonreversals. For example, think of a very familiar conjunction-say, RED APPLE- which is very likely to be prestored. The instance that a subject retrieves in judging this concept may well be the same one he/she would retrieve in response to APPLE. As a result, no typicality reversal is expected and no typicality reversal should occur. Situations like this-ones in which the salience of properties is roughly the same in the simple concept and corresponding complex one-are more likely to occur with familiar than with unfamiliar complex concepts (Smith \& Osherson, 1984). Another way to look at it is to note that the instance-retrieval hypothesis leads us to expect a greater dependence of typicality reversals on instance retrieval for familiar than for unfamiliar complex concepts, but not a greater number of typicality reversals for familiar than for unfamiliar concepts.

We note that the lesser dependence of typicality reversals on instance retrieval for the unfamiliar concepts, coupled with the larger total number of reversals for the same concepts, suggests that some processes other than instance retrieval may be operating. Thus, we have some evidence that conceptual combination may indeed be noncompositional. Importantly, the evidence is not subject to the interpretation that the noncompositionality is due to retrieval of instances from memory, because many of the reversals cannot be predicted from the instances. We will return to the noncompositionality issue in the General Discussion.

One factor that may have influenced the present results concerns the timing of the tasks in Experiment 2. The subjects were asked to retrieve instances for all concepts before being asked the questions about target properties that were used to predict the typicality reversals. As noted earlier, this procedure was employed to ensure that subjects did not neglect to retrieve instances once they realized that only one piece of information was of interest to the experimenter. However, by temporally separating instance generation from the propertyjudgment phase, we made it possible that the answers to the questions were not based on judgments about the retrieved instances. We therefore need to demonstrate that the correlation between predicted and observed typicality reversals remains when questions about target properties are asked at the time of instance generation. Experiment 3 fulfills this goal.

\section{EXPERIMENT 3}

In Experiment 3, we attempted to ensure that subjects base their answer to each target question solely on information in a retrieved instance by asking subjects the question right after they had described the instance. Subjects were interviewed individually by an experimenter in order to ensure that they did, indeed, retrieve an instance for each concept and that they then used that instance to answer the target question.

\section{Method}

Materials. Six sheets containing the concepts and questions from Experiment 2 were prepared (each sheet corresponded to a booklet in Experiment 2). There were four sheets with 16 complex concepts each, one with 18 simple concepts, and one with 19 simple concepts.

Subjects. Seventy-eight University of Michigan students participated for pay. Thirteen subjects retrieved instances for each set of concepts.

Procedure. The subjects were tested individually. Instructions were similar to those of Experiment 2, except that now the subjects were told to retrieve and describe just one instance for each concept. They were further instructed that the experimenter would sometimes ask them a question about the instance. The subjects were given up to $60 \mathrm{sec}$ to describe each instance, but usually took less than $30 \mathrm{sec}$. If the subjects retrieved only a property, they were asked if they were thinking of a particular instance that had the mentioned property (these types of responses were rare). If the subjects retrieved more than one instance, they were asked to describe the first one that had come to mind. When the subjects indicated that they had finished describing an instance, the question about the target property was asked (if the information had not been given during the description). Many of the questions were open-ended-for example, for SPOON, the question was, What was it made of? (In Experiment 2, the corresponding question had 
been, Is it made of metal or wood?). Each subject received the concepts in a different random order.

\section{Results and Discussion}

We used the same simple procedure here as we had before to predict the percentage of subjects in Experiment 1 who would exhibit typicality reversals in a particular direction. The predicted percentage of reversals is given in the last column of the Appendix. The correlation between predicted and obtained reversals was .28 $(d f=62, p<.05)$. Again, the correlation was higher for the many-instances-retrieved concepts than for the fewinstances-retrieved concepts (.35 vs. .23 ). Although this difference is not significant, only the first is significantly greater than $0(d f=30, p<.05)$. There is no difference between the correlations for familiar and unfamiliar concepts ( $r=.32$ and $r=.33$, respectively), and both correlations are significantly greater than $0(d f=30, p<$ $.05)$. Although the overall correlation is significant, there is reason to be concerned about the stability of the present result. Because open-ended questions were often used in Experiment 3, the answer to the target question did not always correspond to one of the choices in the typicality task. This, combined with the relatively small subject group sizes (13 subjects assigned to each set or sheet of concepts), resulted in several predicted reversals' being based on the judgments of a small number of subjects. If we redo the prediction of reversals using only concepts for which 10 or more subjects answered the question with one of the choices in the typicality task, the correlation between predicted and obtained reversals (using all instances retrieved) rises to $.53(d f=39$, $p<.01$ ). Again, the difference between correlations for the many-instances-retrieved and those for the fewinstances-retrieved concepts was in the expected direction, but not significant. However, only the correlation for the many-instances-retrieved concepts was significantly greater than $0(r=.67, d f=19 p<.01$, vs. $r=$ $.37, d f=20, p>.05$ ). Again, there was no significant difference between the correlations for familiar and unfamiliar concepts $(r=.59$ and $r=.52$, respectively). These results generally support the instance-retrieval hy pothesis.

\section{GENERAL DISCUSSION}

\section{Instance Retrieval and Conceptual Combination}

The most important claim of the instance-retrieval hypothesis is that typicality reversals may be due to the retrieval of instances from prestored complex concepts. This claim leads to the prediction that there should be a positive correlation between typicality reversals, on the one hand, and the salience of certain properties when subjects simply describe stored instances of complex concepts, on the other. This prediction was strongly supported in our studies; we consistently found substantial correlations between typicality reversals obtained in Experiment 1 and reversals predicted on the basis of the salience of target properties in retrieved instances. By supporting the instance-retrieval hypothesis, these results show the viability of exemplar models of categorization for natural categories, which has rarely been demonstrated before.

Our correlations were consistently higher for concepts for which subjects could retrieve many instances than for concepts for which they could retrieve few instances. Additionally, the correlations were sometimes higher for concepts that were judged familiar than for those judged unfamiliar. These results also call into question the previous evidence for conceptual combination's being noncompositional. Because many of the concepts used in the prior work were familiar- that is, concepts for which instances could be retrieved easily - the evidence may not reflect aspects of conceptual combination, per se. The evidence may in fact be evidence that instance retrieval is not compositional.

Because the critical results are correlations, one may question whether instance retrieval truly causes typicality reversals. A skeptic might claim that contrary to our assumptions, the instance-retrieval task is actually a conceptual combination task in disguise, and that consequently the correlation of interest merely reflects that we are measuring the same processes by two different means. That is, on each trial of the instance-retrieval tasks, subjects may have composed a complex concept from the two constituent concepts, and then judged the typicality of the two test properties vis à vis the complex concept. This scenario seems highly unlikely. For one thing, it assumes that subjects in our instance-retrieval tasks completely ignored our instructions and instead performed a task that, on the face of it, seems more difficult than the one we asked them to do. Secondly, the scenario in question seems particularly implausible for Experiment 2, in which no target properties were even presented until all instances had been retrieved. In such circumstances, the instances that subjects generated could not be influenced by the target properties, which makes this task very different in structure from the typicality judgment task. Furthermore, it would be difficult for the skeptic to maintain that instance retrieval is not occurring during the typicality task, given that, in Experiment 2 , the correlation between predicted and obtained reversals was very highly dependent on the number of instances that subjects retrieved. In light of arguments such as these, it seems reasonable to assume that our instance-retrieval tasks in fact measure what they purport to measure.

\section{Compositionality Reconsidered}

Earlier we noted that (1) although there are more typicality reversals with unfamiliar complex concepts than with familiar ones, (2) the dependence of typicality reversals on item retrieval may be less for the unfamiliar concepts. Taken together, these two facts imply that mechanisms other than instance retrieval were responsible for some of the typicality reversals with unfamiliar concepts. These other mechanisms might well involve reasoning about causal, theoretical, and other plausible 
relations between concepts (e.g., Medin \& Shoben, 1988; Murphy, 1988; Wisniewski \& Gentner, 1991), which could involve information other than that contained in the constituent concepts. Hence, these mechanisms might result in true violations of compositionality. While we began our analysis by questioning the evidential basis for inferring violations of compositionality, our results suggest that such violations do occur, particularly with unfamiliar concepts. Clearly, future research can explore these potential noncompositional conceptual combination processes.

Note, though, that even if some noncompositional mechanisms are involved, they may come into play relatively late in the processing, after compositional processes have provided a first pass at conceptual combination. This assumption is explicit in the selective modification model of Smith et al. (1988). In this model, a complex concept like BROWN APPLE is constructed by modifying the noun concept by just those properties that are denoted by the adjective (hence the concept BROWN APPLE is identical to APPLE except on the color attribute). However, the possibility is left open for subsequent noncompositional processes that rely on world knowledge rather than just the information contained in the constituent concepts. Other researchers have raised similar suggestions (e.g., Springer \& Murphy, 1992). One piece of evidence for such a hybrid model is the finding that when the latencies of typicality judgments are surreptitiously recorded, typicality reversals are more likely to occur on slower trials (Smith \& Gray, 1990).

\section{REFERENCES}

HaMPTON, J. A. (1987). Inheritance of attributes in natural concept conjunctions. Memory \& Cognition, 15, 55-71.

Heit, E., \& Barsalou, L. W. (1992). An instantiation model of category typicality and instability. In Proceedings of the Fourteenth Annual Conference of the Cognitive Science Society (pp. 360-365). Hillsdale, NJ: Erlbaum.

Medin, D. L., \& SCHAFFER, M. M. (1978). A context theory of classification learning. Psychological Review, 85, 207-238.

Medin, D. L., \& Shosen, E. J. (1988). Context and structure in conceptual combination. Cognitive Psychology, 20, 158-190.

MurPHy, G. L. (1988). Comprehending complex concepts. Cognitive Science, 12, 529-562.
Roth, E. M., \& Shoben, E. J. (1983). The effect of context on the structure of categories. Cognitive Psychology, 15, 346-378.

Smith, E. E., \& GRaY, K. C. (1990). Mechanisms of conceptual combination. Paper presented at the meeting of the Midwestern Psychological Association, Chicago.

Smith, E. E., \& Osherson, D. N. (1984). Conceptual combination with prototype concepts. Cognitive Science, 8, 337-361.

Smith, E. E., Osherson, D. N., Rips, L. J., \& Keane, M. (1988). Combining prototypes: A selective modification model. Cognitive Science, 12, 485-527.

SPRINGER, K., \& MURPhy, G. L. (1992). Feature availability in conceptual combination. Psychological Science, 3, 111-117.

WISNIEWSKI, E. J., \& GENTNER, D. (1991). On the combinatorial semantics of noun pairs: Minor and major adjustments to meaning. In G. B. Simpson (Ed.), Understanding word and sentence (pp. 241284). Amsterdam: Elsevier, North-Holland.

\section{NOTES}

1. We use capitals to indicate concepts.

2. This is not to say that investigators neglect the familiarity of concepts entirely. For example, Springer and Murphy (1992) had an independent group of subjects rate their complex concepts for farmiliarity. Taken at face value, the results indicated that the complex concepts of particular interest were unfamiliar. But there is reason to be skeptical. The rating task included, as fillers, some very familiar complex concepts, such as DIET SODA, and these might have increased the perceived unfamiliarity of the critical complex concepts.

3. The booklets also contained an additional 28 items that are not relevant to the concerns of this paper.

4. Specifically, 15 subjects rated the 64 complex concepts' familiarity. The concepts were presented on a single page, in one of two random orders. The subjects were asked to rate how familiar the concepts were on a scale of 0 to 10 (higher numbers indicating more familiar). BLUE DOG was given as an example of a concept that would probably be judged as unfamiliar. The range of average familiarity ratings obtained for the 64 concepts was 0.27 to 9.4 . Classification into high- and low-familiarity groups was based on a median split. The 32 familiar concepts had a mean rating of $7.70(S D=1.04)$. The 32 unfamiliar concepts had a mean rating of $2.10(S D=1.06)$.

5. In retrospect, mentioning the strategy of "constructing an image of the instance" seemed a mistake because it might have encouraged subjects to engage in constructive processes akin to conceptual combination when trying to describe instances of truly novel complex concepts. However, it appears that subjects rarely used this strategy. We examined all instance descriptions and counted the number of cases in which the described object seemed imaginary. Only 37 such cases were found among a total of 1,372 instance descriptions (a single coder did the examination). Although this estimate includes only those instances that are obviously imaginary, the very small number of positive cases suggests that the vast majority of described instances were, indeed, retrieved from memory rather than constructed. 


\section{APPENDLX \\ Concepts, Properties, Reversals Obtained and Predicted: Experiments 1-3}

The first property listed for a concept in the table below was the one that was most frequent among the instances generated for the simple concept. For example, a majority of instances of BALL in Experiment 2 were hard.

The entries in the column headed Total \% Reversals Observed correspond to the total number of subjects who reversed typicality ordering when going from simple to complex concept (reported in Experiment 1).

The entries in the column headed Directional \% Reversals Observed correspond to the number of subjects in Experiment 1 who reversed typicality in the predicted direction (according to instances retrieved in Experiment 2). For example, $68 \%$ of subjects reversed from "hard" > "soft" for BALL to "soft" > "hard" for LARGE BALL. The total number of reversals for a particular concept is often higher because it includes subjects who reversed in the opposite direction. When the correlations reported in Experiment 2 are recomputed using the (Total) \% Reversals obtained, the conclusions do not change. For example, the correlations between predicted and obtained reversals for the "many instances retrieved" and "few instances retrieved" concepts are $r=.72$ and $r=.23$, respectively (using all instances retrieved). This difference between correlations is significant $(Z=$ $2.55, p<.01)$.

\begin{tabular}{|c|c|c|c|c|c|c|}
\hline \multirow[b]{2}{*}{ Concepts } & \multirow[b]{2}{*}{ Properties } & \multirow[b]{2}{*}{$\begin{array}{c}\text { Total \% } \\
\text { Reversals } \\
\text { Observed } \\
\end{array}$} & \multirow[b]{2}{*}{$\begin{array}{c}\text { Directional } \\
\% \text { Reversals } \\
\text { Observed }\end{array}$} & \multicolumn{3}{|c|}{ Directional \% Reversals Predicted } \\
\hline & & & & $\begin{array}{c}\text { All } \\
\text { Instances } \\
\text { Exp. } 2\end{array}$ & $\begin{array}{c}\text { First } \\
\text { Instance } \\
\text { Exp. } 2\end{array}$ & Exp. 3 \\
\hline \multicolumn{7}{|l|}{ Familiar } \\
\hline LARGE BALL & $\mathrm{hard} / \mathrm{soft}$ & 68 & 68 & 24 & 45 & 12 \\
\hline SMALL BALL & hard/soft & 23 & 5 & 5 & 5 & 8 \\
\hline LARGE BIRD & sings/crows & 91 & 91 & 63 & 63 & 83 \\
\hline SMALL BIRD & sings/crows & 9 & 5 & 4 & 17 & 17 \\
\hline LARGE BOAT & diesel engine/sail & 59 & 0 & 20 & 38 & 25 \\
\hline HARDCOVERED BOOK & fiction/text & 59 & 59 & 11 & 8 & 5 \\
\hline PAPERIBACK BOOK & fiction/text & 36 & 0 & 16 & 31 & 36 \\
\hline LARGE PRINT BOOK & fiction/text & 55 & 32 & 3 & 32 & 60 \\
\hline WHITE CLOUD & sunny day/rainy day & 91 & 0 & 28 & 10 & 56 \\
\hline GRAY CLOUD & sunny day/rainy day & 9 & 9 & 46 & 90 & 44 \\
\hline THORNY FLOWER & garden/wild & 59 & 50 & 12 & 20 & 2 \\
\hline CLEAR CIEM & expensive/cheap & 27 & 14 & 12 & 15 & 8 \\
\hline SHORT GRASS & green/brown & 32 & 32 & 13 & 7 & 8 \\
\hline DARK-COLORED SHIRT & summer/winter & 62 & 59 & 38 & 34 & 14 \\
\hline LICHT-COL.ORED SHIRT & summer/winter & 38 & 0 & 6 & 20 & 100 \\
\hline L.ARGIE SPOON & metal/wooden & 64 & 59 & 3 & 27 & 8 \\
\hline SMALL SPOON & metal/wooden & 18 & 5 & 13 & 0 & 0 \\
\hline GRAVEI. STREET & empty/busy & 73 & 0 & 19 & 13 & 58 \\
\hline PAVED STREET & empty/busy & 23 & 18 & 18 & 19 & 21 \\
\hline LARCGE TELEVISION & color/black and white & 5 & 5 & 10 & 7 & 15 \\
\hline SMALL TELEVISION & color/black and white & 86 & 86 & 49 & 47 & 69 \\
\hline WALL CALENDAR & illustrated/plain & 24 & 9 & 17 & 12 & 0 \\
\hline DRESS SOCK & white/black & 77 & 77 & 65 & 79 & 82 \\
\hline BROWN GLOVES & cloth/leather & 14 & 17 & 5 & 4 & 38 \\
\hline WEEKLYY NEWSPAPER & thick/thin & 36 & 14 & 5 & 10 & 35 \\
\hline METAL DESK & flat/tilted & 0 & 0 & 7 & 1 & 8 \\
\hline (HALKBOARD ERASER & pink/black & 68 & 68 & 62 & 50 & 80 \\
\hline HALLOWEEN CANDY & unhealthy/nutritious & 0 & 0 & 2 & 7 & 8 \\
\hline BEER BOTTLE & clear/colored & 71 & 50 & 33 & 27 & 46 \\
\hline BOTTLED WATER & odorless/aromatic & 0 & 0 & 12 & 22 & 23 \\
\hline J.EGAL PAD & white/yellow & 64 & 59 & 44 & 32 & 38 \\
\hline BUSINESS ENVELOPE & rectangular/square & 9 & 5 & 1 & 0 & 0 \\
\hline
\end{tabular}


APPENDIX (Continued)

\begin{tabular}{|c|c|c|c|c|c|c|}
\hline \multirow[b]{2}{*}{ Concepts } & \multirow[b]{2}{*}{ Properties } & \multirow[b]{2}{*}{$\begin{array}{c}\text { Total \% } \\
\text { Reversals } \\
\text { Observed }\end{array}$} & \multirow[b]{2}{*}{$\begin{array}{c}\text { Directional } \\
\% \text { Reversals } \\
\text { Observed }\end{array}$} & \multicolumn{3}{|c|}{ Directional $\%$ Reversals Predicted } \\
\hline & & & & $\begin{array}{c}\text { All } \\
\text { Instances } \\
\text { Exp. } 2\end{array}$ & $\begin{array}{c}\text { First } \\
\text { Instance } \\
\text { Exp. } 2\end{array}$ & Exp. 3 \\
\hline \multicolumn{7}{|l|}{ Unfamiliar } \\
\hline SQUARE BALL & hard/soft & 32 & 14 & 10 & 21 & 2 \\
\hline CRAWLING BIRD & sings/crows & 68 & 68 & 31 & 50 & 17 \\
\hline LEAF-EATING BIRD & sings/crows & 41 & 36 & 21 & 17 & 26 \\
\hline SEATLESS BOAT & diesel engine/sail & 36 & 23 & 18 & 29 & 4 \\
\hline TRIANGULAR BOOK & fiction/text & 32 & 5 & 12 & 42 & 27 \\
\hline STRIPED FLOWER & garden/wild & 50 & 45 & 6 & 2 & 21 \\
\hline SOFT GEM & expensive/cheap & 62 & 59 & 54 & 63 & 22 \\
\hline ORANGE GEM & expensive/cheap & 82 & 77 & 63 & 100 & 51 \\
\hline CAVE GRASS & green/brown & 91 & 91 & 14 & 17 & 80 \\
\hline UNDERWATER GRASS & green/brown & 41 & 41 & 7 & 8 & 8 \\
\hline PLASTIC SHIRT & summer/winter & 57 & 32 & 24 & 100 & 100 \\
\hline ANIMAL'S SHIRT & summer/winter & 52 & 48 & 18 & 4 & 56 \\
\hline WHITE SPOON & metal/wooden & 41 & 27 & 9 & 50 & 0 \\
\hline INDESTRUCTIBLE SPOON & metal/wooden & 23 & 0 & 10 & 14 & 8 \\
\hline GRASS STREET & empty/busy & 77 & 0 & 3 & 13 & 33 \\
\hline ROUND TELEVISION & color/black and white & 50 & 50 & 25 & 39 & 18 \\
\hline SOLAR-POWERED TELEVISION & color/black and white & 32 & 32 & 25 & 22 & 4 \\
\hline RECTANGULAR WATCH & dial/digital & 59 & 41 & 1 & 17 & 25 \\
\hline WINTER TOMATO & juicy/dry & 68 & 68 & 33 & 33 & 40 \\
\hline ASYMMETRIC CHAIR & wood/plastic & 55 & 55 & 34 & 31 & 3 \\
\hline WOOL SHOES & black/red & 32 & 27 & 8 & 25 & 25 \\
\hline THREE-WHEELED CAR & gas powered/electric powered & 91 & 91 & 19 & 10 & 11 \\
\hline SQUARE CANTALOUPE & sweet/bland & 36 & 36 & 8 & 19 & 9 \\
\hline WOODEN BICYCLE & ten speed/one speed & 95 & 95 & 46 & 69 & 51 \\
\hline BENT CORN & yellow/blue & 14 & 14 & 3 & 7 & 0 \\
\hline BLUE FUR & genuine/imitation & 76 & 68 & 54 & 44 & 67 \\
\hline YELLOW PEAS & round/oval & 23 & 23 & 4 & 15 & 23 \\
\hline FLAT PIE & homemade/storebought & 77 & 68 & 0 & 4 & 0 \\
\hline SANDLESS BEACH & hot/cold & 59 & 59 & 30 & 35 & 32 \\
\hline GLASS BASKET & useful/decorative & 59 & 50 & 45 & 91 & 44 \\
\hline PAPER ROPE & long/short & 77 & 71 & 33 & 36 & 13 \\
\hline
\end{tabular}

(Manuscript received June 13, 1994; accepted for publication August 9, 1994.) 